\title{
Influence of Having Breakfast on Happiness and Attention-Concentration among High School Students
}

\author{
S. Marriyah Irfan and S. Fatimah Irfan
}

\section{ABSTRACT}

\begin{abstract}
Background: Healthful nutrition has a positive connection with both physical and mental health. Breakfast is often considered as the most important meal of the day. Despite the benefits, skipping the morning meal is highly prevalent among adolescents. Healthy eating has an effect on happiness and concentration but a firm connection has not been established. Breakfast skipping can be a factor and a gap in the literature exists in this regard. The aim of this study was to evaluate the relationships between eating breakfast, happiness and attention concentration among high school students.
\end{abstract}

Methods: In this descriptive cross-sectional study, a convenient sampling strategy was used to select the students from an International school located in Riyadh, Saudi Arabia. The 29-item Oxford Happiness Questionnaire along with demographic variables was employed as research instrument. Chi-square test, student t-test and ANOVA were used to compare different categorical variables.

Results: The overall Happiness mean score was $3.621 \pm 0.6637$. Students who were regular at having breakfast every day had higher happiness mean scores. Overall, $40 \%$ of the participants reported to have decreased concentration/attentiveness during the first 4 lectures at school. Majority $(55 \%)$ of the breakfast skippers were distracted in the lessons. A regular breakfast eating habit was found to be significantly associated with happiness $(p=0.04)$ and concentration during lectures $(p=0.04)$ respectively. Majority of the students had insufficient physical activity $(72 \%)$ and had experienced stress $(88 \%)$ in the last six months respectively. The prevalence of being overweight/ obese among breakfast skippers was higher $(39 \%)$ than was in breakfast consumers $(26 \%)$.

Conclusion: Eating breakfast was associated with higher happiness scores and better concentration among high school students. An alarmingly high prevalence of stress among students is a cause of concern. Schools should start breakfast eating programs and promote physical activity to reduce stress. Future studies are needed to explore the factors associated with stress.

Keywords: happiness, breakfast, attention-concentration, high school.
Submitted : March 17, 2021

Published : April 07, 2021

ISSN: 2593-8339

DOI: $10.24018 /$ ejmed.2021.3.2.775

\section{S. Marriyah Irfan*}

Pakistan International School English Section (PISES) Riyadh, Saudi Arabia. (e-mail: s.marriyah2003@gmail.com) S. Fatima Irfan

Pakistan International School English Section (PISES) Riyadh, Saudi Arabia. (e-mail: s.fatimahirfan04@gmail.com)

*Corresponding Author

\section{INTRODUCTION}

During recent decades, the happiness and psychological well-being of adolescents have gained a lot of attraction as they play a core role in education and help students flourish in their educational journey as well as build resilience against adversity. As future contributors to society, a healthy present status of pupils is essential. According to the World Health Organization (WHO), health is a state of general physical, psychological, and social well- being [1].

Literature suggests that happiness and well- being of students is related to their performance and creativity. Happiness is characterized by positive feelings such as optimism, positive thinking, and the perception of personal well-being and stress is an experience that is emotionally and physiologically challenging. The experience of stress, whether academic or social; acute or chronic has deleterious effects on mental health and physical health outcomes leading to poor health practices [2]. A plethora of research shows that students all over the world, in higher education, are facing high rates of depression and anxiety [3].

This affects pupils' health and academic performance. Various factors are linked with school performance such as physical activity, obesity, stress and dietary habits [4]. Breakfast consumption is emphasized by parents and teachers and it is considered as an important meal of the day, and a prerequisite for successful learning after the long fasting period without uptake of energy and nutrients. Skipping breakfast and extending the fasting period has been associated with many adverse consequences due to metabolic changes such as worse mood, increased risk of obesity and poor cognitive function [5]. By regularly skipping morning meals, individuals are at an increased risk of atherosclerosis, heart disease, high blood pressure, diabetes and high cholesterol leading to poor cardiometabolic health [6]-[9]. Breakfast is required to restore glycogen and stabilize insulin 
levels [10]. Consuming breakfast also has a positive effect on cortisol. Cortisol levels are highest early morning, around 7 am; breakfast consumption drops the level. Skipping breakfast blunts this typical drop effect. Therefore, it is essential to eat something to bring the hormone levels down as it can disrupt body processes leading to chronic stress and a feeling of anxiety [11].

Despite the physical and mental benefits of eating breakfast, skipping the morning meal is highly prevalent. From an international perspective, a significant decrease in daily breakfast consumption is noticed among adolescents [12]. Across the world, girls and older school-age children are found to skip breakfast more frequently as compared to their counterparts. Numerous reasons for skipping breakfast were given, including the lack of time in the morning, no desire to eat food or body weight control especially by females [13]. Skipping breakfast was reported in $17 \%-52 \%$ of those aged 13-18 years and is a proxy factor for unhealthy lifestyle [5], [14]. The proportion of breakfast skippers among adolescents in Saudi Arabia is estimated around 10-40\% [15].

Most of the factors that contribute to happiness are past one's authority. However, what individuals eat is within their control. Many studies have evaluated the state of happiness and its related factors, but literature on the association between breakfast and happiness is scarce. It is important to find out how eating habits can alter the mood and affect happiness. The aim of the present study was to evaluate the relationships between happiness plus concentration in the first four lectures at school and breakfast eating in a group of female high school students in an International school in Riyadh, Saudi Arabia.

Research Questions: What role does breakfast play in happiness and concentration?

\section{Hypothesis:}

It was hypothesized that the students having regular breakfast will have a higher happiness score than their counterparts. Furthermore, students having regular breakfast will have better attention during class.

\section{MATERIALS AND METHODS}

\section{A. Study Design and Setting}

It was a cross sectional descriptive study and targeted a group of high school students in an International school, Riyadh, Saudi Arabia during the academic year 2019-2020. Like other Saudi schools, it operates on a single-gender basis (teaches the two genders separately). It was a fairly homogeneous set of students with similar ethnicity and cultural backgrounds.

\section{B. Sampling}

The good calculators' website was used to estimate the required sample size. The sample size (n) was calculated according to the formula:

$$
\mathrm{n}=\left[\mathrm{z}^{2} \times \mathrm{p} \times(1-\mathrm{p}) / \mathrm{e}^{2}\right] /\left[1+\left(\mathrm{z}^{2} \times \mathrm{p} \times(1-\mathrm{p}) /\left(\mathrm{e}^{2} \times \mathrm{N}\right)\right)\right.
$$

where: $\mathrm{z}=1.96$ for a confidence level of $95 \%, \mathrm{p}=0.5, \mathrm{~N}=$ 219 , margin of error $(e)=0.05$.

Based on the assumptions the estimated sample size was 140. The present study was part of the science project and was approved by the school Ethics Committee. The necessary permissions were obtained from Education Office and school authorities.

\section{Data Collection}

A convenient sample selection was applied. All students who were attending the high school were invited to participate in the study. Participation was voluntary. The principal investigator SMI had a meeting with students of each class in order to explain the purpose of the study. An informed verbal consent was received from the interested participants. A short demographic questionnaire (six items) was developed to gather information from the participants. Happiness was measured by the Oxford Happiness Questionnaire. Breakfast eating was assessed by asking how often they eat breakfast. Skipping breakfast was defined as sometimes or never having breakfast [16]. Food habits were assessed by a multiple choice on diet pattern. Other factors for skipping breakfast were assessed by asking questions on dieting to lose weight, stress experience in the last 6 months and physical activity. The level of physical activity was assessed by a multiple-choice question. Responses were categorized as Sedentary (no physical activity), Light and regular activity (activities that do not produce sweat or shortness of breath), Insufficient activity (completing less than two-three hours of moderate intensity physical activity each week), Sufficient activity (2-3 hours or more of moderate or vigorous activity per week). Ratings of concentration/alertness were also collected from the participants as: Distracted, slightly distracted, Attentive, Fully attentive during the first 4 lectures. The data collection lasted around two weeks.

\section{Measuring Instrument: The Oxford Happiness Questionnaire $(\mathrm{OHQ})$}

Happiness is defined as something that is on our mind and it can be measured using questioning [17]. The OHQ inventory is a 29-item self-report instrument for measuring well-being and personal happiness with six response options ranging from 1 to 6 for each item. The score ranges from minimum 29- total maximum 174, where higher scores indicate a higher level of happiness. A score of 1-2: not happy, 2-3: somewhat unhappy, 3-4: not particularly happy or unhappy, 4-5: rather happy; pretty happy and 5-6: very happy, 6: too happy. This questionnaire was selected because of its wide use and its high reliability and improved concurrent and content validity and broad applicability for research.

\section{E. Quality Assurance}

The questionnaires along with a covering letter, highlighting the aims and objectives of the study, were distributed to the students in person by the principal investigator. Students returned the completed questionnaires either immediately or after the class finished.

\section{F. Statistical Analysis}

All statistical analyses were performed using SPSS version 21 (IBM Corp, Armonk, NY). Descriptive statistics along with Chi-square test were used to compare the happiness scores and other socio-demographic factors. A p value of less than 0.05 was considered significant. 


\section{Results}

Out of a total of 140 students, 100 participated in the study and filled out the printed questionnaire ( $71 \%$ response rate). The mean age of the participants was $16.16 \pm 0.918$ years (age range 14-18 years). Table I shows frequency of demographic characteristics and key variables of the students. The mean of total happiness score was $3.62 \pm 0.663$ ( $\min 1.8$, $\max 4.9$ ). The mean \pm standard deviation of the total happiness score among regular breakfast consumers was $3.70 \pm 0.66$, while the mean \pm standard deviation of total happiness score among breakfast skippers was $3.42 \pm 0.63$. The overall prevalence of breakfast skippers (sometimes or never/week) was $31 \%$ and less than half (49\%) had breakfast almost every day (Table II). Habitually eating breakfast was found to be significantly associated with happiness $(\mathrm{p}=0.04)$ (Fig. 1).

\begin{tabular}{|c|c|}
\hline Variable & Number $(\%)$ \\
\hline \multicolumn{2}{|l|}{ Study year } \\
\hline Grade 10 & 24 \\
\hline Grade 11 & 53 \\
\hline Grade 12 & 23 \\
\hline \multicolumn{2}{|l|}{ Weight } \\
\hline$<40 \mathrm{KG}$ & 2 \\
\hline $41-60$ & 74 \\
\hline$>60$ & 24 \\
\hline \multicolumn{2}{|l|}{ Self-reported weight } \\
\hline Low weight & 10 \\
\hline Normal & 60 \\
\hline Overweight & 25 \\
\hline Obese & 5 \\
\hline \multicolumn{2}{|l|}{ Self-reported physical activity } \\
\hline Sedentary & 16 \\
\hline Light and regular activity & 44 \\
\hline Insufficient activity & 28 \\
\hline Sufficient activity & 12 \\
\hline \multicolumn{2}{|l|}{ Stress experience in last 6 months } \\
\hline Yes & 88 \\
\hline No & 12 \\
\hline \multicolumn{2}{|l|}{ How often do you have breakfast } \\
\hline Never & 10 \\
\hline Sometimes & 21 \\
\hline Often & 20 \\
\hline Always & 49 \\
\hline \multicolumn{2}{|l|}{ Your concentration during the first 4 lectures } \\
\hline Distracted & 7 \\
\hline slightly distracted & 33 \\
\hline Attentive & 51 \\
\hline fully attentive & 9 \\
\hline \multicolumn{2}{|l|}{ Your diet pattern } \\
\hline breakfast lunch dinner & 21 \\
\hline breakfast snack lunch snack dinner & 43 \\
\hline snack lunch snack dinner & 23 \\
\hline snacks and dinner & 11 \\
\hline only snacks & 2 \\
\hline \multicolumn{2}{|l|}{ Happiness score } \\
\hline 1-2: Not happy & 2 \\
\hline 2-3: Somewhat unhappy & 15 \\
\hline $\begin{array}{l}\text { 3-4: Not particularly happy or } \\
\text { unhappy }\end{array}$ & 58 \\
\hline 4-5 Rather happy & 25 \\
\hline 5-6: Very happy, & 0 \\
\hline 6: Too happy & 0 \\
\hline
\end{tabular}

A correlation between breakfast consumption and concentration amongst the students was also observed. Consumption of breakfast showed positive effects on the concentration of the study population. Overall, $40 \%$ of the participants reported to have decreased concentration/attentiveness during the first 4 lectures in the school. Majority $(55 \%)$ of the breakfast skippers were distracted in the lessons, compared to $33 \%$ of regular breakfast-eaters. A statistically significant association was found between regular breakfast eating and concentration during lectures ( $\mathrm{p}=0.04)$ (Fig. 2; Table II).

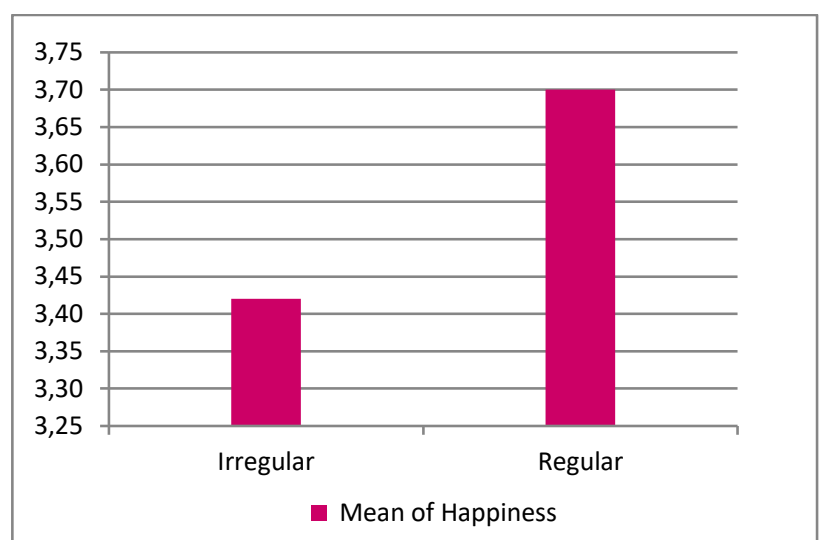

Fig.1. Association between Happiness score and breakfast consumption among students.

TABLE II: ASSOCIATION BETWEEN BREAKFAST EATING AND SOCIODEMOGRAPHIC CHARACTERISTICS AMONG HIGH SCHOOL STUDENTS

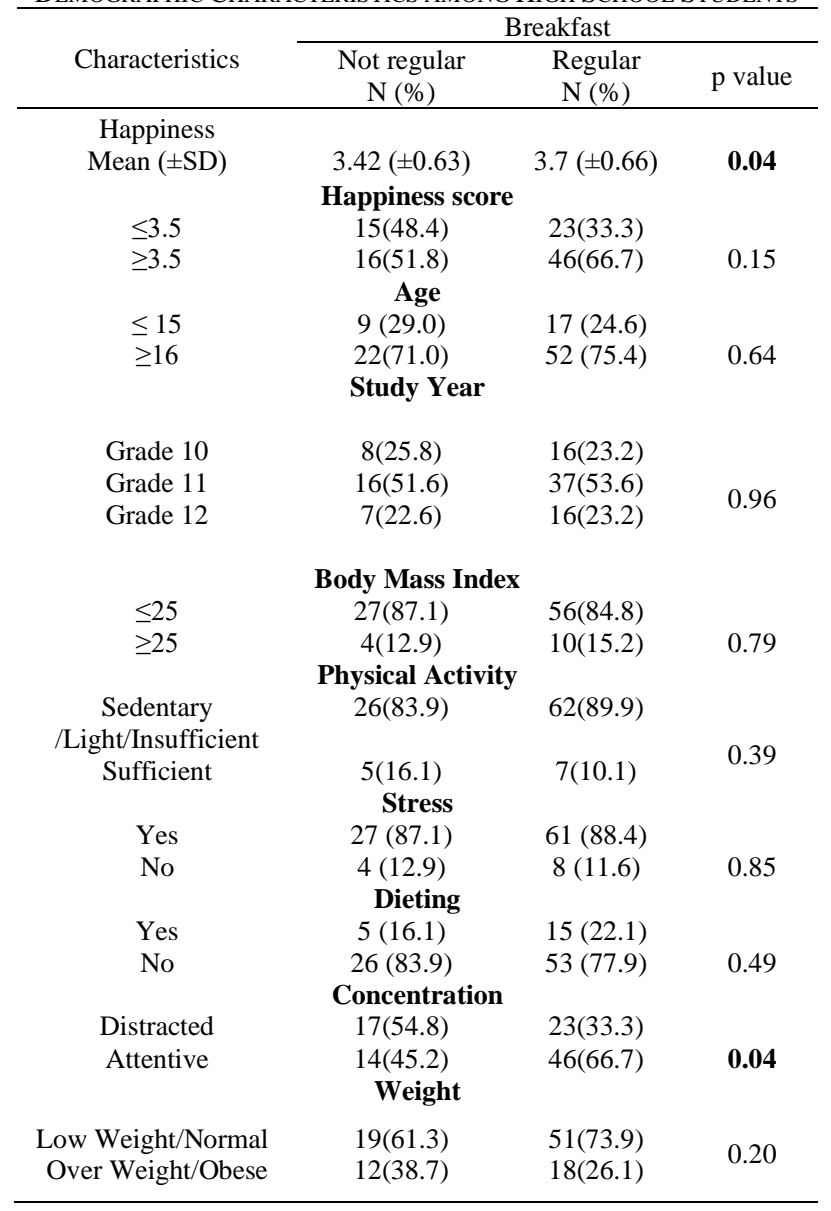

Majority of the students reported to experience stress in the last six months (88\%) and insufficient physical activity (88\%) (Table I).

The prevalence of being overweight and obesity amongst breakfast skippers was higher (39\%) than breakfast consumers $(26 \%)$, but this difference was not statistically significant $(\mathrm{p}=0.20)$ (Fig. 3). No significant association was found between students' age, study year, physical activity, dieting, BMI/weight, stress and the breakfast consumption. 


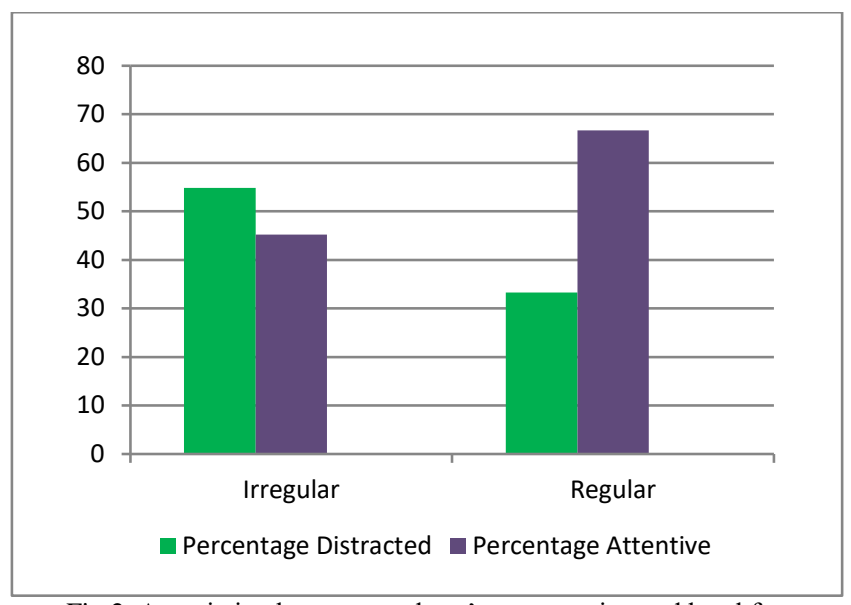

Fig.2. Association between students' concentration and breakfast consumption.

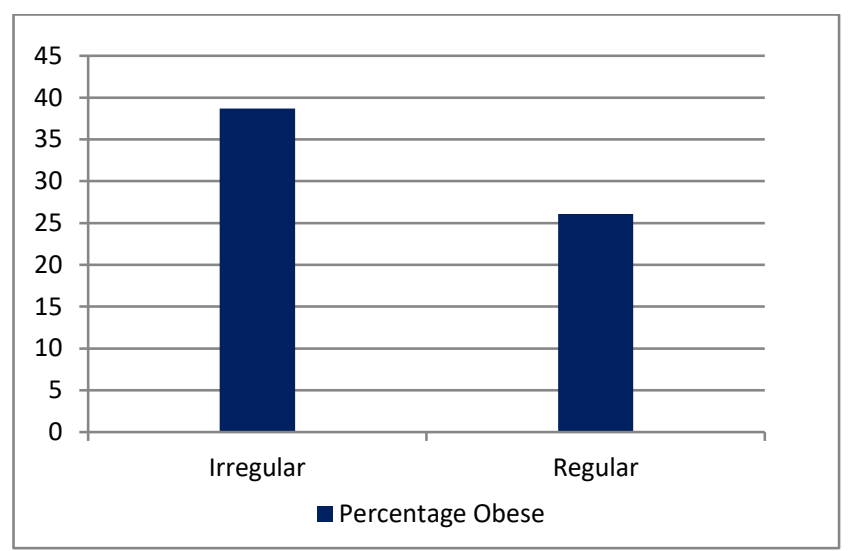

Fig. 3. Obesity and breakfast eating.

\section{DISCUSSION}

Breakfast skipping is common during middle adolescence and young adulthood, which is a period of transition and development. With a gradually increasing number of these breakfast skippers, there is a need to evaluate whether skipping of breakfast affects the mood and concentration of these fasting students. To the best of our knowledge, this is one of the first studies on breakfast consumption and happiness among high school girls in the Arab region. An alarmingly low number of participants were rather happy, and none were very happy; which is a cause of concern. The findings clarify that there is a significant relationship between breakfast consumption, happiness, and cognitive performance (attention/concentration), which is in line with the hypothesis as well as previous studies [5], [18], [19]. A further intervention and research exploring the causes is needed.

Breakfast is breaking a fasting period from the day before and therefore the most crucial meal to provide energy for all the activities that lay ahead. The gap of about ten to twelve hours between dinner and breakfast causes low blood glucose levels. Habitually missing breakfast can adversely affect wellbeing and cognitive performance and thus highlights its importance. In our sample, $31 \%$ of the high school girls were breakfast skippers, which is within the range and in line with the large-scale surveys in the western region, where $18-36 \%$ of adolescents skip this important meal [20]. It is notable to find in this study that happiness scores were less among breakfast skippers as compared to their counterparts.

This variation could be explained in part by different mechanisms that can have an effect on the overall outcome. Firstly, the effect of family time in having breakfast together can have a positive effect on an individual's mood early in the morning. Secondly, at a metabolic level, breakfast can give the energy reserve for the day ahead. It helps to restore glycogen and stabilize insulin levels, and this certainly does help the mood. Thirdly, on a psycho-biological level, the macronutrients eaten during breakfast can influence mood by acting on brain chemistry and neurotransmitters [21]. Systematic reviews demonstrate the role of diet in modulating wellbeing, and a positive effect of breakfast consumption on cognition and mood within a few hours post ingestion as well as less depression [22]. As theoretically expected, and in agreement with previous studies, the present study findings suggest that not having breakfast was detrimental and had a bad influence on attention-concentration during the morning session of the school. Breakfast skippers usually feel hungry in the morning sessions but eat later in the day to catch up. This may be associated with snacking and the consumption of fast foods of poor nutritional value. On the contrary, breakfast eaters have a higher basal metabolic rate and less food cravings [23]. Furthermore, the snacking dietary pattern among participants of the study is of concern because of their potential for increasing risk for developing various types of ill-health issues [24].

Overall, the findings from the current study shed light on the hither-to unexplored relationship between breakfast eating, stress, happiness, physical activity, and obesity among high school students. The prevalence of being overweight and obese was significantly lower amongst breakfast consumers (26\%) than breakfast skippers $(39 \%)$. This finding is in line with the reported literature [25]-[27]. Furthermore, a majority of the students reported to experience stress and insufficient physical activity respectively. The question arises that is it because of the bi-directional relationship; stressed students engaging in unhealthy behaviors such as skipping meals, less physical activity leading to obesity or vice versa? Because the contributing factors were not investigated in depth, this gap opens an opportunity for more research surrounding this relationship.

Another possible explanation could be overweight or obese students tend to skip their breakfast meal as an intentional weight loss strategy. However, literature shows that avoidance from eating at proper meal times will create an energy deficit; causing overeating later in the day, resulting in weight gain [28]. During breaktime, pupils usually go for fast food or a glucose drink which are typically very substandard in terms of nutrition and cause certain irreparable effects on a person's health; such as obesity which is an emerging major public health problem globally among adolescents [29].

Literature has shown that skipping breakfast impairs attention and memory, and this impairment increases in magnitude during the morning hours [22], [28]. Skipping might cause low energy, lethargy, less daily physical and mental activities and mood swings. This study provides some initial evidence that breakfast has a positive effect and a 
proper meal in the morning may help students to function better cognitively and psychosocially [30].

Future work should explore the association of sociocultural, behavioral, and ecological risk factors with breakfast consumption among adolescents. Schools should provide youth with healthy eating opportunities. Study findings have implications for school management and suggest steps that schools could take to encourage healthier eating practices among students by starting School Breakfast Programs. Campaigns which focus on key components like schools' food quality, participation in meal programs, physical activity, physical education, and nutrition education that make healthy and active individuals should be introduced and executed. This study should also be conducted in different school populations to provide further insight.

\section{STRENGTH AND Limitations}

This study was the first to assess the relationship between breakfast and happiness among high school students using a widely used inventory. From an applied perspective, this research indicates an opportunity for school health personnel to gain new insight. However, it is not without limitations including the use of self-administered inventories rather than structured interviews and involving only a single school. This study was cross-sectional, so causal relationships cannot be drawn. The study was carried out with students from an international school in Riyadh and inclusion of other schools could have resulted in different effects. Future studies should be directed to find out the reasons at the student level, exploring the main reasons for skipping breakfast such as lack of time, stress, lack of morning appetite and concern about their body weight.

\section{FUNDING}

The authors received no specific funding for this research.

\section{AUTHOR'S CONTRIBUTIONS}

SMI was involved in the concept, study design, literature review, manuscript writing and data analysis. SFI was involved in literature review, data acquisition and manuscript critical review and editing. The authors believe that the manuscript represents honest work, read the final draft and approved.

\section{ACKNOWLEDGMENT}

The authors express their gratitude to all the students who participated in the study and the teachers who gave support during the project. This research could not have been conducted in the absence of their cooperation. We would like to give special thanks to Dr. Ambreen Kazi, Princess Nora Bent Abdullah Chair for Women's Health Research, King Saud University, for her tremendous help and input in the statistical analysis of the results. We are forever indebted to our dear mother, Dr. Farhana Irfan for her guidance that enabled us to humbly produce this unique research.

\section{REFERENCES}

[1] Lesani, Azedah, Mohammad Asghar, Maryam Javadi, and Ali Fakhari. “"Eating Breakfast, Fruit and Vegetable Intake and Their Relation with Happiness in College Students." "Eating and weight disorders 21, no. 4 (December 1, 2016): 645-51. https://doi.org/10.1007/s40519-0160261-0.

[2] Kolehmainen, Matthew A Stults, and Raita Sinha. "The Effects of Stress on Physical Activity and Exercise." Sports Med 44, no. 1 (January 2014): 81-121. https://doi.org/10.1007/s40279-013-0090-5.

[3] Kolehmainen, Matthew A Stults, and Raita Sinha. "The Effects of Stress on Physical Activity and Exercise." Sports Med 44, no. 1 (January 2014): 81-121. https://doi.org/10.1007/s40279-013-0090-5.

[4] Kim, So Young, Songyong Sim, Bumjung Park, Il Gyu Kong, JinHwan Kim, and Hyo Geun Choi. "Dietary Habits Are Associated With School Performance in Adolescents.” Medicine 95, no. 12 (March 2016): e3096. https://doi.org/10.1097/md.0000000000003096.

[5] Wadolowska, Lidia, Jadwiga Hamulka, Joanna Kowalkowska, Natalia Ulewicz, Magdalena Gornicka, Marta Jeruszka-Bielak, Małgorzata Kostecka, and Agata Wawrzyniak. "Skipping Breakfast and a Meal at School: Its Correlates in Adiposity Context. Report from the ABC of Healthy Eating Study of Polish Teenagers." Nutrients 11, no. 7 (July 11, 2019): 1563. https://doi.org/10.3390/nu11071563.

[6] Uzhova, Irina, Valentín Fuster, Antonio Fernández-Ortiz, José M. Ordovás, Javier Sanz, Leticia Fernández-Friera, Beatriz López-Melgar, et al. "The Importance of Breakfast in Atherosclerosis Disease." Journal of the American College of Cardiology 70, no. 15 (October 10, 2017): 1833-42. https://doi.org/10.1016/j.jacc.2017.08.027.

[7] Cahill, Leah E., Stephanie E. Chiuve, Rania A. Mekary, Majken K Jensen, Alan J. Flint, Frank B. Hu, and Eric B. Rimm. "Prospective Study of Breakfast Eating and Incident Coronary Heart Disease in a Cohort of Male US Health Professionals." Circulation 128, no. 4 (July 23, 2013): 337-43. https://doi.org/10.1161/circulationaha.113.001474.

[8] Watanabe, Yoko, Isao Saito, Ikuyo Henmi, Kana Yoshimura, Kotatsu Maruyama, Kanako Yamauchi, Tatsuhiro Matsuo, et al. "Skipping Breakfast Is Correlated with Obesity." Journal of Rural Medicine 9, no. 2 (June 17, 2014): 51-58. https://doi.org/10.2185/jrm.2887.

[9] Geliebter, Allan, Nerys M. Astbury, Roni Aviram-Friedman, Eric Yahav, and Sami Hashim. "Skipping Breakfast Leads to Weight Loss but Also Elevated Cholesterol Compared with Consuming Daily Breakfasts of Oat Porridge or Frosted Cornflakes in Overweight Individuals: a Randomised Controlled Trial." Journal of Nutritional $\begin{array}{lllll}\text { Science } & 3 & \text { (November } 13, & \text { 2014): }\end{array}$ https://doi.org/10.1017/jns.2014.51.

[10] Adolphus, Katie, Clare L. Lawton, and Louise Dye. "The Effects of Breakfast on Behavior and Academic Performance in Children and Adolescents." Frontiers in Human Neuroscience 7 (August 8, 2013): 425. https://doi.org/10.3389/fnhum.2013.00425.

[11] Witbracht, Megan, Nancy L. Keim, Shavawn Forester, Adrianne Widaman, and Kevin Laugero. "Female Breakfast Skippers Display a Disrupted Cortisol Rhythm and Elevated Blood Pressure." Physiology \& Behavior 140 (March 1, 2015): 215-21. https://doi.org/10.1016/j.physbeh.2014.12.044.

[12] Zakrzewski, J K, F B Gillison, S Cumming, T S Church, P T Katzmarzyk, S T Broyles, C M Champagne, et al. "Associations between Breakfast Frequency and Adiposity Indicators in Children from 12 Countries." International Journal of Obesity Supplements 5, no. S2 (December 5, 2015): 80-88. https://doi.org/10.1038/ijosup.2015.24.

[13] Rampersaud, Gail C., Mark A. Pereira, Beverly L. Girard, Judi Adams, and Jordan D. Metzl. "Breakfast Habits, Nutritional Status, Body Weight, and Academic Performance in Children and Adolescents.' Journal of the American Dietetic Association 105, no. 5 (May 2005): 743-60. https://doi.org/10.1016/j.jada.2005.02.007.

[14] Adolphus, Katie, Clare L Lawton, Claire L Champ, and Louise Dye. "The Effects of Breakfast and Breakfast Composition on Cognition in Children and Adolescents: A Systematic Review." Advances in Nutrition 7, no. 3 (May 16, 2016): 590S-612S. https://doi.org/10.3945/an.115.010256.

[15] Sami H Alzahrani, Abdulmajeed Abdulaziz Saeedi, Maan Khaleed Baamer, Abdullah Faisal Shalabi, Abdullah M Alzahrani "Eating Habits Among Medical Students at King Abdulaziz University, Jeddah, Saudi Arabia". International journal of general medicine 13 (Mar 2020):77-88. https://doi.org/ 10.2147/IJGM.S246296.

[16] Wardle, J, A M Haase, and A Steptoe. "Body Image and Weight Control in Young Adults: International Comparisons in University Students from 22 Countries." International Journal of Obesity 30, no. 4 (April 6, 2006): 644-51. https://doi.org/10.1038/sj.ijo.0803050.

[17] Veenhoven, Ruut. "Will Healthy Eating Make You Happier? A Research Synthesis Using an Online Findings Archive." Applied 
Research in Quality of Life, August 14, 2019. https://doi.org/10.1007/s11482-019-09748-7.

[18] Piqueras, José A, Walter Kuhne, Pablo Vera-Villarroel, Annemieke van Straten, and Pim Cuijpers. "Happiness and Health Behaviours in Chilean College Students: A Cross-Sectional Survey.” BMC Public Health 11, no. 1 (June 7, 2011): 443. https://doi.org/10.1186/14712458-11-443.

[19] Allgöwer, Annette, Jane Wardle, and Andrew Steptoe. "Depressive Symptoms, Social Support, and Personal Health Behaviors in Young Men and Women.” Health Psychology 20, no. 3 (May 2001): 223-27. https://doi.org/10.1037/0278-6133.20.3.223.

[20] Pengpid, Supa, and Karl Peltzer. "Skipping Breakfast and Its Association with Health Risk Behaviour and Mental Health Among University Students in 28 Countries." Diabetes, Metabolic Syndrome and Obesity: Targets and Therapy Volume 13 (August 18, 2020): 2889-97. https://doi.org/10.2147/dmso.s241670.

[21] Antonio Affinita, Loredana Catalani, Giovanna Cecchetto, Gianfranco De Lorenzo, Dario Dilillo, Giorgio Donegani, Lucia Fransos, Fabio Lucidi, Chiara Mameli, Elisa Manna, Paolo Marconi, Giuseppe Mele, Laura Minestroni, Massimo Montanari, Mario Morcellini, Giuseppe Rovera, Giuseppe Rotilio, Marco Sachet and Gian Vincenzo Zuccotti." Breakfast: a multidisciplinary approach". Italian Journal of Pediatrics Vol 39 (Jul 10,2013); 44. http:/doi.org/ 10.1186/1824-7288-39-44.

[22] Ferrer-Cascales, Rosario, Miriam Sánchez-SanSegundo, Nicolás RuizRobledillo, Natalia Albaladejo-Blázquez, Ana Laguna-Pérez, and Ana Zaragoza-Martí. "Eat or Skip Breakfast? The Important Role of Breakfast Quality for Health-Related Quality of Life, Stress and Depression in Spanish Adolescents." International Journal of Environmental Research and Public Health 15, no. 8 (August 19, 2018): 1781. https://doi.org/10.3390/ijerph15081781.

[23] Gajre, Nitin S, Sylvia Fernandez- Rao, Shahnaz Vazir, and Nagalla Balakrishna. "Breakfast Eating Habit and Its Influence on AttentionConcentration, Immediate Memory and School Achievement." Indian Paediatrics 45, no. 10 (October 17, 2008): 824-28.

[24] Neumark-Sztainer, Dianne, Simone A French, Peter J Hannan, Mary Story, and Jayne A Fulkerson. "School Lunch and Snacking Patterns among High School Students: Associations with School Food Environment and Policies." The international journal of behavioral nutrition and physical activity 6, no. 1 (October 6, 2005): 14 . https://doi.org/10.1186/1479-5868-2-14.

[25] Catherine S Berkey, Helaine RH Rockett, M W Gillman, Alison E Field, and Graham A Colditz. "Longitudinal Study of Skipping Breakfast and Weight Change in Adolescents." International Journal of Obesity 27, no. 10 (October 26, 2003): 1258-66. https://doi.org/10.1038/sj.ijo.0802402.

[26] Vanelli, Maurizio, Brunella Iovane, Anna Bernardini, Giovanni Chiari, Maria Katrin Errico, Chiara Gelmetti, Matteo Corchia, Anna Ruggerini, Elio Volta and Stefano Rossetti. "Breakfast Habits of 1,202 Northern Italian Children Admitted to a Summer Sport School. Breakfast Skipping Is Associated with Overweight and Obesity'." Acta bio-medica: Atenei Parmensis 76, no. 2 (October 2005): 79-85.

[27] Timlin, Maureen T., Mark A. Pereira, Mary Story, and Diane NeumarkSztainer. "Breakfast Eating and Weight Change in a 5-Year Prospective Analysis of Adolescents: Project EAT (Eating Among Teens)." PEDIATRICS 121, no. 3 (March 1, 2008): e638-45. https://doi.org/10.1542/peds.2007-1035.

[28] Wesnes, Keith A, Claire Pincock, David Richardson, Gareth Helm, and Simon Hails. "Breakfast Reduces Declines in Attention and Memory over the Morning in Schoolchildren." Appetite 41, no. 3 (December 2003): 329-31. https://doi.org/10.1016/j.appet.2003.08.009.

[29] Bahadoran, Zahra, Parvin Mirmiran, and Fereidoun Azizi. "Fast Food Pattern and Cardiometabolic Disorders: A Review of Current Studies." Health Promotion Perspectives 5, no. 4 (January 30, 2016): 231-40. https://doi.org/10.15171/hpp.2015.028.

[30] Spence, Charles. "Breakfast: The Most Important Meal of the Day?" International Journal of Gastronomy and Food Science 8 (July 2017): 1-6. https://doi.org/10.1016/j.ijgfs.2017.01.003. 